\title{
„Wer hat Angst vor Jack Ma?“ \\ Heroisierung und Deheroisierung eines chinesischen Unternehmerhelden
}

\section{Nicola Spakowski / Jennifer Anchali Stapornwongkul}

Der chinesische Unternehmer Jack Ma, Gründer und Geschäftsführer des chinesischen Unternehmens Alibaba, wird in der westlichen Presse vielfach als „Held“ tituliert $(19,43)$. Wo der Heldenbegriff nicht fällt, wird aus dem weiteren semantischen Feld von Berühmtheit geschöpft, und er wird als „Star“ (49, 11), „Weltstar“ $(38,71)$ oder „celebrity“ $(12,57)$ bezeichnet. Er und sein Unternehmen versammeln Superlative und internationale Auszeichnungen auf sich und nehmen in globalen Rankings einen vorderen Platz ein. ${ }^{1}$ Jack Ma, geboren 1964 und in China als Ma Yun bekannt, stellt damit ein aussagekräftiges Beispiel für Strategien der Heroisierung und Deheroisierung in interkultureller Perspektive dar. ${ }^{2}$

Im vorliegenden Beitrag verstehen wir Jack Ma als ,Unternehmerhelden' - ein auf den österreichischen Ökonomen Joseph A. Schumpeter (1883-1950) zurückgehendes Konzept - und untersuchen das Bild Jack Mas in der westlichen, vor allem der deutschen Presse seit ca. 2014. ${ }^{3}$ Wir beabsichtigen, die Grundzüge dieses Bildes aufzuzeigen, die Faktoren zu identifizieren, die dieses Bild prägen, und die Strategien der Heroisierung und Deheroisierung zu benennen, die in der Berichterstattung über Jack Ma vorkommen. Ganz grundsätzlich möchten wir zeigen, dass das Bild Jack Mas changiert zwischen einem (positiv konnotierten) Repräsentanten des global vorkommenden Unternehmerhelden und dem (negativ konnotierten), spezifisch chinesischen Gesicht eines autoritären Kapitalismus. Diese Ambivalenz in der Wahrnehmung Jack Mas entspricht - so unsere These - der ambivalenten Haltung zu China allgemein, die zwischen Bewunderung und Schrecken oszilliert. Die Einpassung Jack Mas in dieses zwiespältige Bild erfolgt vor allem über Projektio-

Siehe Teil 3 dieses Beitrags.

2 Dieser Beitrag befasst sich ausschließlich mit der westlichen und speziell deutschen Perzeption Jack Mas. Die chinesische Perzeption folgt völlig eigenen Mustern. So wird Jack Ma im chinesischen Internet beispielsweise „Papa Ma Yun“ (Ma Yun baba) genannt, siehe Pan Xianghui / Yang Peng: „Ma Yun baba“. Shuzi shidai de yingxiong chongbai yu fensi jiamian. Yi zhong chuanbo shehuixue fenxi („Papa Ma Yun“. Heldenverehrung und Fankult im Zeitalter des Internets. Eine sozialkommunikative Analyse), in: Tansuo yu zhengming 9, 2018, S. 65-75. Siehe auch David J. Davies: China's Celebrity Entrepreneurs. Business Models for ,Success', in: Louise Edwards / Elaine Jeffreys (Hg.): Celebrity in China, Hong Kong 2010, S. 193-216. Die vertiefte Analyse der Perzeption Jack Mas in China bleibt einer späteren Untersuchung vorbehalten.

3 Diesem Beitrag liegt die Analyse von 75 Zeitungsartikeln zugrunde. Dieses Quellenkorpus, das bemerkenswert einförmig und repetitiv ist, ist geeignet, Grundlinien der Perzeption Jack Mas aufzuzeigen. Ein Anspruch auf Vollständigkeit besteht nicht. 
nen: Die Eigenschaften eines bestimmten Typus - der Unternehmerheld; das chinesische System oder der chinesische Nationalcharakter - werden auf Jack Ma übertragen, bzw. umgekehrt: Jack Ma repräsentiert den jeweiligen Typus und verleiht ihm ein Gesicht. Als typischer Unternehmerheld erscheint er - den heroischen Pendants des Silicon Valley vergleichbar - als autonomer Akteur mit einem individuellen Charakter, der ,Chinas Aufstieg' im positiven Sinne verkörpert: als Aufholprozess, in dem China eine westliche Entwicklungslogik verbürgt und gleichzeitig neue Investitionsmöglichkeiten eröffnet. Als typischer chinesischer Unternehmer wird er hingegen im chinesischen Kontext verortet und mit den angeblichen Eigenschaften des chinesischen Systems verknüpft: einem autoritären, intervenierenden Staat, einem aggressiven Expansionsstreben und der Produktpiraterie. Sowohl Individualität als auch Autonomie werden ihm in dieser Perspektive abgesprochen.

Dieser ambivalente Zuschnitt der Darstellung Jack Mas erklärt sich auch aus aktuellen Zeitdiagnosen und wirtschaftspolitischen Perspektiven: dem ,Aufstieg Chinas und der sich verschärfenden Konfrontation zwischen China und den USA, die gerade auf dem Feld innovativer Technologien ausgetragen wird, und der Digitalisierung mit ihrem disruptiven Potenzial. Es sind genau diese sich überlagernden Krisen bzw. Felder des Wettbewerbs, die einen chinesischen Internetunternehmer wie Jack Ma überhaupt erst in den westlichen Blick rücken. Dieser westliche Blick ist gleichzeitig national gefärbt, denn verschiedene Staaten nehmen in den genannten Konflikt- und Konkurrenzfeldern unterschiedliche Positionen ein, die auch die Diskussion ökonomischer und politischer Problemlagen beeinflussen. Wir sind zwar vorsichtig, unsere Beobachtungen als ,deutsche' Perzeption Jack Mas zu klassifizieren, nehmen in den deutschen Zeitungsartikeln aber doch Gewichtungen und Sensibilitäten wahr, die von der englischsprachigen Presse leicht abweichen. Unseres Erachtens hat Jack Ma in Deutschland eine bessere Presse als im englischsprachigen Raum, wo das chinesische Wirtschaftssystem deutlich kritischer gesehen wird. Selbst wenn die deutsche Berichterstattung zu China gegenwärtig immer negativer wird, überwiegen hier doch die positiven Darstellungen Jack Mas.

Der Beitrag ist viergegliedert. Der erste Teil stellt das Konzept des Unternehmerhelden vor und benennt übliche Heroisierungsstrategien - und, davon abgeleitet, auch Strategien der Deheroisierung -, wie sie auch auf Jack Ma Anwendung finden. Gleichzeitig zeigen wir hier die Verbindung des Unternehmerhelden zu spezifischen ökonomischen Idealen und Innovationsstrategien auf, die zentral sind für die Konfliktlagen, welche in das Bild Jack Mas hineinspielen. Im zweiten Teil geht es um das ambivalente China-Bild in deutschen Medien, das auf Jack Ma ein eher positives oder negatives (oder ambivalentes) Licht wirft. Wir gehen hier auf die Zusammenhänge zu übergeordneten Vorstellungen der internationalen Ordnung ein - Globalismus versus Realismus - und verwenden neben dem Begriff der ,Deheroisierung' auch den der ,Diskreditierung', um zu zeigen, mit welchen Strategien nicht allein die Person, sondern das gesamte System angegriffen werden. Die 
beiden einleitenden Teile begründen die Benennung von Heroisierungs- und Deheroisierungs- bzw. Diskreditierungsstrategien des konkreten Beispiels Jack Ma, wie sie in den Teilen 3 und 4 dargelegt werden.

\section{Schumpeters Unternehmerheld im Kontext der Innovationsproblematik}

Helden sind, in Anlehnung an die Definition des Freiburger Sonderforschungsbereichs „Helden - Heroisierungen - Heroismen“, „basal über Außerordentlichkeit, besondere Handlungsmacht und Agonalität" bestimmt. ${ }^{4}$ Wird diese Definition an die westliche Berichterstattung zu Jack Ma angelegt, so repräsentiert er eindeutig einen ,Helden': durch die Außerordentlichkeit, wie sie in Rankings, quantitativen Superlativen und dem ihm zugeschriebenen Charakter zum Ausdruck kommt; durch die Handlungsmacht als Unternehmer; und durch die Agonalität seines Handelns, sei es in Auseinandersetzung mit dem Staat und der Bürokratie Chinas, sei es im Wettstreit mit westlichen Internetunternehmen. ${ }^{5}$ Gleichzeitig verkörpert Jack Ma aber auch einen spezifischen Heldentypus, nämlich den Unternehmerhelden, wie er zu Beginn des 20. Jahrhunderts von dem österreichischen Nationalökonomen Joseph Schumpeter ,entdeckt ${ }^{\varsigma}$ und in seinen Persönlichkeitsmerkmalen charakterisiert wurde. ${ }^{6}$

In seiner Theorie der wirtschaftlichen Entwicklung (1912) versuchte Schumpeter, die Dynamik des ökonomischen Prozesses zu erklären, da sie in der Wirtschaftstheorie seiner Zeit zu wenig berücksichtigt wurde. Schumpeter befand Innovation als die Triebkraft des Kapitalismus und den Unternehmer als „Agens“7 dieser Entwick-

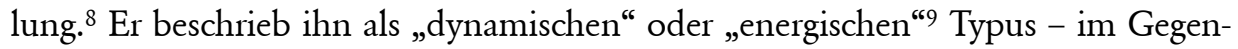
satz zur „Überzahl der statisch disponierten Wirtschaftssubjekte“10 - und als

4 Achim Aurnhammer / Barbara Korte: Einleitung, in: dies. (Hg.): Fremde Helden auf europäischen Bühnen (1600-1900) (Helden - Heroisierungen - Heroismen 5), Würzburg 2017, S. 9-19, hier S. 13. Siehe auch Tobias Schlechtriemen: The Hero and a Thousand Actors. On the Constitution of Heroic Agency, in: Barbara Korte u. a. (Hg.): Heroes and Things. Heroisches Handeln und Dinglichkeit, in: helden. heroes. héros. E-Journal zu Kulturen des Heroischen 4.1, 2016, S. 17-32, DOI: 10.6094/helden.heroes.heros./2016/01/03 sowie die vielen Veröffentlichungen im Rahmen des Sonderforschungsbereichs unter www.sfb 948.uni-freiburg.de/.

5 Siehe die Ausführungen in den Teilen 3 und 4.

6 Auch Werner Sombart hat zum Bild des heroischen Unternehmers beigetragen, siehe Georg Kohler: Händler, Unternehmer, Kapitalist und Manager - zur Typologie des Wirtschaftsmenschen, in: Ludger Heidbrink / Peter Seele (Hg.): Unternehmertum. Vom Nutzen und Nachteil einer riskanten Lebensform, Frankfurt/New York 2010, S. 27-42, hier S. 34-37. Zur Figur des Unternehmerhelden und dem postheroischen Management siehe auch Ulrich Bröckling: Postheroische Helden. Ein Zeitbild, Berlin 2020, S. 141-163. Joseph Schumpeter: Theorie der wirtschaftlichen Entwicklung, Leipzig 1912, S. 147.

8 Siehe v. a. Kap. 2, „Das Grundphänomen der wirtschaftlichen Entwicklung“, ebd., S. 103198.

9 Ebd., S. 128.

10 Ebd., S. 131. 
„Mann der Tat", 11 der etwas Neues (ein neues Produkt, eine neue Produktionsmethode, einen neuen Markt) schafft oder erschließt und dabei kein Risiko scheut. Diesen Unternehmertypus zeichnen nach Schumpeter Persönlichkeitsmerkmale aus wie Dynamik, Kreativität, Tatkraft und Risikobereitschaft - einschließlich des Muts zu scheitern. Wenn im heutigen Wirtschaftsjournalismus Innovation und die besondere Form von Start-up-Unternehmen behandelt werden, bestehen oft direkte oder indirekte Bezüge zu Schumpeters Typus des Unternehmerhelden.

Eine neue, kritische Unternehmerforschung legt die rhetorischen Mittel frei, mit denen Unternehmer in den Medien und sogar der Fachliteratur als Unternehmerhelden in Schumpeters Sinne konstruiert werden. ${ }^{12}$ Für unseren Zusammenhang bedeutend ist dabei zunächst die narrative Sequenzierung des Werdegangs eines Unternehmers in Form der sogenannten Heldenreise. ${ }^{13}$ Dieser Begriff geht auf den amerikanischen Literaturwissenschaftler Joseph Campbell zurück, der die ,Heldenreise' als universales mythologisches Motiv beschrieb. In seinem Essay The Entrepreneur as Hero (1999) überträgt Candace A. Allen die ,Heldenreise' griffig und mit Bewunderung auf das Unternehmertum:

The first stage involves departure from the familiar and comfortable into the unknown, risking failure and loss - a venturing forth for some greater purpose or idea. The second stage is the encountering of hardship and challenge, and the mustering of courage and strength to overcome or discover. The third is the return to the community with something new or better than what was there before. Ultimately, the hero is the representative of the new [...]. What I will contend here is that in our modern world, the wealth creators - the entrepreneurs - actually travel the heroic path and are every bit as bold and daring as the heroes who fought dragons or overcame evil. ${ }^{14}$

Die kritische Unternehmerforschung benennt weitere Kennzeichen der medialen Repräsentation von Unternehmern. Ganz grundsätzlich geht sie davon aus, dass die Medien aus Unternehmern legitime soziale Akteure machen..$^{15}$ Auch das spezielle ökonomische Kalkül eines Unternehmens spiele eine Rolle: Das Bild des Unternehmers wird zum „marketing tool“16 und ist wichtiger Teil der Botschaft, die an

11 Der Begriff wird vielfach verwendet, z. B. ebd., S. 10.

12 Alistair R. Anderson / Lorraine Warren: The Entrepreneur as Hero and Jester. Enacting the Entrepreneurial Discourse, in: International Small Business Journal 29.6, 2011, S. 589-609. John O. Ogbor: Mythicizing and Reification in Entrepreneurial Discourse. IdeologyCritique of Entrepreneurial Studies, in: Journal of Management Studies 37.5, 2000, S. 605635.

13 Joseph J. Pilotta: The Entrepreneur as Hero?, in: Vicente Berdayes / John W. Murphy (Hg.): Neoliberalism, Economic Radicalism, and the Normalization of Violence, Cham u. a. 2016, S. 37-52.

14 Candace A. Allen: The Entrepreneur as Hero, in: Economic Insights 2.1, 1999, ohne Paginierung. Eine kritische Einschätzung der unternehmerischen Heldenreise findet sich bei Ogbor: Mythicizing (Anm. 12), S. 617.

15 Miruna Radu / Renaud Redien-Collot: The Social Representation of Entrepreneurs in the French Press. Desirable and Feasible Models?, in: International Small Business Journal 26.3, 2008, S. 259-298, hier S. 261.

16 Anderson / Warren: Entrepreneur (Anm. 12), S. 591. 
potenzielle Investoren ausgesendet wird, welche sich gerade im Falle von Start-ups nicht an vergangenen Leistungen orientieren können. ${ }^{17}$ Für den Wirtschaftslaien verliehen Unternehmer der Ökonomie ein Gesicht - „the friendly face of capitalism" 18 -, und sie machten komplexe ökonomische Vorgänge nachvollziehbar: „The concept of entrepreneurship is fuzzy, entrepreneurial process is complex and often intangible, but entrepreneurs are real people. ${ }^{\text {"19 }}$ Komplexitätsreduktion liegt insbesondere dort vor, wo der Unternehmer als ,an atomistic and isolated agent of change" konstruiert wird, unter Ausblendung des Milieus „that supports, drives,

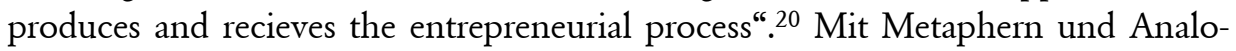
gien werden unbekannte Unternehmen und Unternehmer an Bekanntes und Vertrautes assimiliert. ${ }^{21}$ In der Fremdcharakterisierung wie Selbstinszenierung von Unternehmern dominiert eine eigenartige Kombination von Typizität und persönlicher Einzigartigkeit. ${ }^{22} \mathrm{Ihr}$ besonderes Profil erhalten sie auch im Kontrast mit Gegenbildern - die Forschung spricht von „contrastive subjects“ und einer „,contrastive strategy". ${ }^{23}$ So hat der schumpetersche Unternehmerheld sein typisches Gegenbild in der Bürokratie, deren Vertreter als „pedantic, inert, unimaginative, uncreative, inflexible, producer-focused, rule-bound“" 24 erscheinen. ${ }^{25}$

Wenn individuelle Agency, Autonomie, Kreativität, Innovation, Risiko und persönliche Eigenart die vorrangigen Eigenschaften des Unternehmerhelden sind, dann wird umgekehrt ein Unternehmer dort deheroisiert, wo er in sein Umfeld eingebettet wird und ihm gegenteilige Eigenschaften und Handlungsweisen zugeschrieben werden: Abhängigkeit, Innovationsschwäche oder Produktpiraterie, Protektion und der Einfluss eines Nationalcharakters.

17 Michael Lounsbury / Mary Ann Glynn: Cultural Entrepreneurship. Stories, Legitimacy, and the Acquisition of Resources, in: Strategic Management Journal 22, 2001, S. 545-564, hier S. 549.

Sarah Drakopoulou Dodd / Alistair R. Anderson: Mumpsimus and the Mything of the Individualistic Entrepreneur, in: International Small Business Journal 25.4, 2007, S. 341360, hier S. 341, in Anlehnung an Frank Bechhofer und Brian Elliot.

Ebd., S. 348.

20 Ebd., S. 342.

21 Lounsbury / Glynn (Anm. 17): Cultural, S. 549.

22 Anderson / Warren: Entrepreneur (Anm. 12); Lounsbury / Glynn: Cultural (Anm. 17), S. 554.

23 Radu / Redien-Collot: Representation (Anm. 15), S. 275.

24 Anderson / Warren: Entrepreneur (Anm. 12), S. 599, nach C. Grey.

25 Je nach nationalem Kontext werden Unternehmer auch mit anderen Gegenbildern kontrastiert, im französischen Fall etwa mit dem Typus des Angestellten. Gerade die Darstellung von Unternehmern in der französischen Presse zeigt, wie stark das Bild von Unternehmern von nationalen Kontexten geprägt ist. Siehe hierzu Radu / Redien-Collot: Representation (Anm. 15). Siehe auch Sophie Golinski / Sebastian Henn: Imperialisten, Spione oder Retter? Zur Charakterisierung von Direktinvestitionen aus Russland, Indien und China in deutschen Tageszeitungen, in: Zeitschrift für Wirtschaftsgeographie 59.1, 2015, S. 1-19, hier S. 11. 
Doch vor allem repräsentiert der Unternehmerheld mit den von Schumpeter benannten Eigenschaften nicht die Wirtschaftswelt im Allgemeinen, sondern steht für eine Marktwirtschaft mit den typischen bottom-up-Aktivitäten der Privatwirtschaft. Insbesondere die USA, die als sogenannte „liberale Marktwirtschaft" ${ }^{\text {"26 }}$ ein Muster der „radical innovation“ aufweist, bei dem Start-up-Unternehmen und Risikokapital eine wichtige Rolle spielen, ${ }^{27}$ hat mit den Heroen des Silicon Valley Leitbilder hervorgebracht, die globale Ausstrahlung besitzen und die Start-upSzenen weltweit befeuern. Am anderen Ende des Spektrums zwischen Markt und Staat als Koordinationsmechanismen befindet sich China, wo Innovation vorrangig durch eine dezidierte Industriepolitik und langfristige Perspektiven vorangetrieben wird. Ein privatwirtschaftlicher Start-up-Unternehmer erscheint vor diesem Hintergrund geradezu als Anomalie. Dass auch die chinesische Regierung Start-ups toleriert und sogar fördert, ${ }^{28}$ ist im Westen wenig bekannt, mit dem Beispiel Alibaba aber gut zu belegen. Für den Staat als Hauptakteur der Innovation ist der 2015 von der chinesischen Regierung aufgelegte Entwicklungsplan „Made in China 2025“ ein gutes Beispiel, „designed to transform China to a more innovative manufacturing economy with high-end production" ${ }^{29}$ Deutschland wiederum ringt gegenwärtig um eine Innovationspolitik, für die verschiedene Optionen bestehen: die spezifisch deutsche Unternehmerkultur mit ihrer Orientierung an stabilen Produktionsbedingungen und Innovation als graduellem Prozess, ${ }^{30}$ die Förderung von Start-ups nach amerikanischem Vorbild und schließlich Anleihen bei der chinesischen Industriepolitik. Vielleicht ist diese offenere Position Deutschlands der Grund dafür, dass in der deutschen Berichterstattung zu Jack Ma bestimmte Elemente fehlen oder weniger ausgeprägt sind als in US-amerikanischen Texten, so v. a. die Verurteilung der Staatsnähe chinesischer Unternehmer. ${ }^{31}$

26 Dieser Begriff stammt aus der Literatur zu Kapitalismusvarianten, wo die „liberale Marktwirtschaft“ der USA der „koordinierten Marktwirtschaft“ Deutschlands gegenübergestellt wird. Siehe hierzu Peter A. Hall / David Soskice (Hg.): Varieties of Capitalism. The Institutional Foundations of Comparative Advantage, Oxford u. a. 2001.

27 Alexander Ebner: Varieties of Capitalism and the Limits of Entrepreneurship Policy. Institutional Reform in Germany's Coordinated Market Economy, in: Journal of Industry Competition and Trade 10.3/4, 2010, S. 319-341, hier S. 322.

28 Siehe z. B. das vom Staatsrat der VR China 2006 aufgelegte National Medium- and LongTerm Program for Science and Technology Development (2006-2020). An Outline, International Telecommunication Union, www.itu.int/en/ITU-D/Cybersecurity/Documents/ National_Strategies_Repository/China_2006.pdf, 29. Januar 2020.

29 Premier Li Keqiang Urges Greater Push for Innovation, The State Council, People's Republic of China, 17. Juni 2015, english.gov.cn/premier/video/2015/06/17/content_28147 5128932167.htm, 22. Januar 2020.

30 Ebner: Varieties (Anm. 27), S. 322.

31 Siehe Kapitel 4. 


\section{Das China-Bild der Medien zwischen Bereunderung und Schrecken}

In den westlichen Medien spielt die ökonomische Entwicklung Chinas eine große Rolle. ${ }^{32}$ Unter dem Schlagwort des „Aufstiegs Chinas“ werden die große Dynamik seiner ökonomischen Entwicklung und seine zunehmende Wirtschaftskraft verfolgt, teilweise mit Bewunderung, teilweise mit Schrecken - und teilweise in einer ambivalenten Kombination von Bewunderung und Schrecken. ${ }^{33}$ Dabei zeigt ein Überblick über die Berichterstattung seit ca. dem Jahr 2000, dass das ökonomische Potenzial Chinas immer höher veranschlagt und China im globalen Wirtschaftsgeschehen eine immer aktivere Rolle zugeschrieben wird. Wurde Chinas ,Aufstieg anfänglich v. a. an den durchschnittlichen Wachstumsziffern des Landes festgemacht, so erscheint China heute als Akteur mit konkreten Eigenschaften und Strategien. ${ }^{34}$ Und war China zunächst generell als Exportmarkt und Produktionsstätte für westliche Unternehmen relevant, so rückte später zunächst das genaue Profil des chinesischen Konsumenten in den Blick, ${ }^{35}$ bevor dann chinesische Unternehmen mit ihren je eigenen Strategien und Gesichtern wahrgenommen wurden. ${ }^{36}$ Für unseren Zusammenhang aufschlussreich ist die Einschätzung der Innovationsfähigkeit Chinas, denn Weltmarktführung kann nur erringen, wer nicht nur kopiert, sondern selbst neue Produkte kreiert. Auch hier ist die Entwicklung eindeutig:

32 Carola Richter / Sebastian Gebauer (Hg.): Die China-Berichterstattung in den deutschen Medien, Berlin 2010, S. 44. Das Bild Chinas in den Medien kann hier nur angerissen werden. Vertiefende Analysen liefern v. a. Richter / Gebauer: China-Berichterstattung, Kai Hafez: Das Chinabild deutscher Medien aus kommunikationswissenschaftlicher Perspektive, in: Carola Richter / Sebastian Gebauer (Hg.): Die China-Berichterstattung in den deutschen Medien, Berlin 2010, S. 237-258, Thomas Heberer: Chinabild und Medienberichterstattung aus politikwissenschaftlicher Perspektive, in: Carola Richter / Sebastian Gebauer (Hg.): Die China-Berichterstattung in den deutschen Medien, Berlin 2010, S. 259-289, Caja Thimm: China im Spiegel der Printmedien - zwischen Verdammung und Überhöhung? Medieninhalte und Expertenperspektiven zur Berichterstattung in Deutschland, in: Friedemann Vogel / JiaWenjian (Hg.): Chinesisch-Deutscher Imagereport. Das Bild Chinas im deutschsprachigen Raum aus kultur-, medien- und sprachwissenschaftlicher Perspektive (2000-2013), Berlin/Boston 2017, S. 29-47, Yi Edward Yang / Xinsheng Liu: The ,China Threat' through the Lens of US Print Media. 1992-2006, in: Journal of Contemporary China 21.76, 2012, S. 695-711, Li Zhang: The Rise of China. Media Perception and Implications for International Politics, in: Journal of Contemporary China 19.64, 2010, S. 233-254.

33 Siehe zum Folgenden Nicola Spakowski: Asia as Future - The Claims and Rhetorics of an ,Asian Century', in: Marc Frey / Nicola Spakowski (Hg.): Asianisms, Regionalist Interactions, and Asian Integration, Singapur 2015, S. 209-236. Die Beobachtungen zu Asien sind auf China übertragbar. Siehe auch Zhang: Rise (Anm. 32).

34 Es ist insgesamt typisch für die Berichterstattung zur Wirtschaft Chinas, dass der Staat und weniger die Unternehmen als Akteur auftritt, siehe Golinski / Henn: Imperialisten (Anm. 25), S. 10.

35 Siehe z. B. Yuval Atsmon / Max Magni: Meet the Chinese Consumer of 2020, in: McKinsey Quarterly, März 2012.

36 Sprechende Beispiele für die letztgenannte Entwicklung sind Wolfgang Hirn: Chinas Bosse, Frankfurt/New York 2018 und Stephan Scheuer: Der Masterplan. Chinas Weg zur Hightech-Weltherrschaft, Freiburg 2018. 
A dramatic change in outside perceptions of China's capacity for innovation can be seen in the evolution of news headlines over just the past several years. What was once a question of whether China can innovate has become one of how it is doing so. (62)

Jack Mas Alibaba ist ein Beispiel für Innovationsfähigkeit, genauso wie Huawei und Xiaomi. Dass diese chinesischen Marken und die Gesichter ihrer Manager im Westen plötzlich sichtbar werden, hat mit dem globalen Ringen um Technologieführerschaft zu tun, bei dem sich die USA und China geradezu einen „tech cold war" liefern (28), während deutsche oder europäische Konkurrenzprodukte fehlen. ${ }^{37}$

$\mathrm{Ob}$ die genannten Entwicklungen eher positiv, als Chance, oder eher negativ, als Bedrohung, wahrgenommen werden, hängt mit weltanschaulichen Grundsätzen zusammen, die auf die Begriffe ,Globalismus' und ,Realismus' gebracht werden können. ${ }^{38}$ Globalisten haben ein positives Verhältnis zur Globalisierung. Sie betonen Interdependenz, Kooperation und Integration als Grundlagen globaler Prosperität und zeichnen win-win-Szenarien, um den weiteren Abbau von Handels- und Investitionsschranken zu legitimieren. Realisten auf der anderen Seite sehen das Weltgeschehen als Ergebnis der Konkurrenz von Nationalstaaten und die Verteilung der Macht zwischen denselben als Nullsummenspiel. Während die Globalisten nun chinesische Entwicklungen und einzelne Akteure an westliche Erfahrungen angleichen, ${ }^{39}$ zeichnen Realisten nicht nur ein Bild von Konkurrenz, sondern auch eines von Andersartigkeit. ${ }^{40}$ China wird hier zum Rivalen und zum systemisch ,Anderen', und die Liste der bedrohlichen Entwicklungen ist lang: die Herausbildung sogenannter national champions - (Staats)unternehmen, die von der chinesischen Regierung mit dem Ziel der globalen Wettbewerbsfähigkeit gefördert werden - und der damit zusammenhängende Vorwurf des ,Staatskapitalismus'; die ökonomische Expansion Chinas, festzumachen an der Übernahme westlicher Firmen und am großen Plan der ,Neuen Seidenstraße; die bereits genannte Strategie ,Made in China $2025^{\circ}$ in Verbindung mit Vorwürfen des unfairen Wettbewerbs; und der zunehmende Autoritarismus des chinesischen Parteichefs Xi Jinping, abzulesen an der von ihm selbst veranlassten Entfristung seiner Amtszeit und an der Einführung des Sozialkreditsystems, welches als Instrument der totalen Überwachung wahrgenommen wird $(20,30,52,63,69)$. In der Summe lautet die Bilanz etwa so:

37 Die Analyse von Thimm: China im Spiegel (Anm. 32), S. 32, ergab, dass die Wirtschaftsberichterstattung zu China 2012 am positivsten im Vergleich zu anderen Themenbereichen war. Gleichzeitig sind Wirtschaftsthemen jedoch auch am häufigsten mit Bedrohungsszenarien durchzogen (z. B. China als Konkurrent, Verdrängung deutscher Unternehmen). Spakowski: Asia as Future (Anm. 33), S. 211-214.

39 Siehe die Beispiele in ebd., S. 23-226.

40 Chengxin Pan: The „China Threat" in American Self-Imagination. The Discursive Construction of Other as Power Politics, in: Alternatives 29, 2004, S. 305-331. 
Dieses Land provoziert politisch und gesellschaftlich, es steht für ein anderes Wirtschafts- und Lebensmodell. Das Menschenbild der Europäischen Union und ihre Vorstellung einer regelbasierten Zusammenarbeit liberaler Demokratien stehen dazu in Konkurrenz. Tatsächlich handelt es sich um eine handfeste Rivalität, die von nun an immer stärker zu spüren sein wird. (45)

Wie sehr diese negative Bilanz auf eine kontrastive und vereinfachende Rhetorik angewiesen ist, zeigt sich allein an der Beschreibung der Europäischen Union, die sich bekanntlich kaum durch ein einheitliches Menschenbild und Politikmodell auszeichnet.

Konkrete Zeitungstexte über Jack Ma lassen eine deutliche Interferenz zwischen China-Bild und der Zeichnung des Unternehmers und seines Betriebes erkennen: Jack Ma bietet die Projektionsfläche für angeblich typische Eigenschaften Chinas. Für die Bewunderer des chinesischen ,Aufstiegs' exemplifiziert er auf Firmenebene, was China in den vergangenen Dekaden als Nation geleistet hat. In der negativen Sichtweise tritt er demgegenüber nicht mehr als autonomer und individueller Akteur in Erscheinung, sondern in Abhängigkeit vom chinesischen Staat und mit angeblich typischen nationalen Charakterzügen. Das negative Bild Jack Mas wird weiter verstärkt, indem China als System und Nation diskreditiert werden - oder umgekehrt: In Jack Ma manifestieren sich Charakterzüge und systemische Kennzeichen, welche die angebliche Bedrohlichkeit des Systems, den negativen Charakter Chinas und foul play als Handlungsprinzip belegen: staatliche Protektion, Intervention und Produktpiraterie. In der deutschen Berichterstattung zu Jack Ma fällt außerdem der Bezug zu dem in Deutschland vielbeachteten System der sozialen Kreditwürdigkeit auf, womit auch die politische Seite des chinesischen Systems thematisiert wird (15, 50, 33, 24).

\section{Die Heroisierung Jack Mas als Unternebmerheld}

Die Heroisierung Jack Mas in den Medien findet vor allem durch einen Abgleich mit den oben genannten Unternehmermerkmalen statt: Außerordentlichkeit, Handlungsmacht, Risikobereitschaft, Mut zum Scheitern und ein individueller Charakter.

Die Außerordentlichkeit Jack Mas wird zuallererst mit Superlativen belegt, die seine Person und das Unternehmen betreffen: Er ist „der reichste Chinese“ (19, siehe auch 1) bzw. „reichster Asiate“ (70), gehört zu den „wichtigste(n) Denker(n) der Welt" (22) und besetzte - so das Magazin Forbes - 2019 den 21. Platz auf der Liste der weltweit reichsten Personen (46). Alibaba stützt sich auf das „weltweit wohl profitabelste Geschäftsmodell im Internethandel“ (53), ist „der weltweit größte Amazon-Konkurrent“ (34) und „one of the world's largest e-commerce businesses“ (46). Alibaba als Unternehmen zeichnet sich durch ein enormes Ausmaß aus, sei es in Bezug auf die Kundenzahl, den Umsatz oder den Unternehmenswert, der den von Amazon und Ebay zusammen übersteigt $(51,14,6)$. Insbesondere der 
„Börsengang mit Weltrekord“ (55) an der New Yorker Börse 2014 hat das Unternehmen und seinen Gründer in den Blickwinkel der medialen Berichterstattung gerückt $(16,40,42)$. Mit diesem Schritt zog Alibaba in den Spitzenkreis der internationalen Tech-Welt ein.

Außerordentlichkeit zeigt sich auch in Jack Mas unternehmerischen Fähigkeiten und seinem Charakter. Der Unternehmer Jack Ma gilt in erster Linie als „Visionär“ $(66,3,54,27,14,29)$. So titelt die WirtschaftsWoche beispielsweise „Jack Ma - Wie ein Visionär Alibaba zum Erfolg führte“ (55), und die Deutsche Welle konstatiert: „Seine an zahlreichen Rückschlägen geschärften Visionen machten aus einem kleinen Internetportal ein global expandierendes E-Commerce- und EntertainmentImperium" (66). Der Erfolg auf der Grundlage visionärer Kraft wird dabei von scheinbar irrationalen Momenten - Mas fachliche Unbedarftheit $(68,39,5,3)$ und das Verlassen auf sein „Bauchgefühl“ $(5,68)$ - nur unterstrichen. Laut Schumpeter ist es jedoch nicht allein die Geschäftsvision, die einen Unternehmer ausmacht, sondern die Tatkraft in deren Verwirklichung. Dem entspricht Jack Ma als „Visionär, der jede Gelegenheit ergreift“ (18). Entsprechende biographische Darstellungen blenden dabei sowohl sein Umfeld - besonders Mitstreiter und Mitarbeiter als auch den Zufall als Erfolgsfaktoren aus.

Heroisch wirkt Ma besonders dort, wo er sich durch Risikobereitschaft und den Mut zum Scheitern auszeichnet und seine Lebensgeschichte in der Aneinanderreihung von Herausforderungen den Verlauf von Campbells Heldenreise annimmt. Das Scheitern auf etablierten Erfolgsrouten - ob in der Schulzeit oder nach dem Studium - ist ein wichtiger Bestandteil seiner unternehmerischen Erfolgsgeschichte $(32,39,49,35)$ : „Von Harvard zehn Mal abgelehnt, machte er doch Karriere" (5). Entscheidend dabei ist seine Beharrlichkeit im Angesicht der Niederlagen. Sein Verhalten ist von starkem „Durchhaltewillen“ (29) geprägt. Er gilt als „Stehaufmännchen“ (32), vereinigt „Risikobereitschaft und Geduld“ (68) sowie die Fähigkeit und Bereitschaft, Fehler zu begehen, um aus ihnen zu lernen (35). In der Summe ging er einen „holprig[en]“ $(36,35,18)$ Weg. Die ARD titelt: „Bei Jack Ma lief's nicht immer rund“ (5). Trotz aller Schwierigkeiten stellt Mas Leben aber eine Erfolgsgeschichte dar: „Vom Englischlehrer zum Internetmilliardär könnte die Überschrift über Jack Mas Lebenslauf heißen“ (56).

Seine heroischen Qualitäten offenbart Jack Ma besonders im Kontrast mit Gegenbildern und in agonalen Konstellationen. Jack Ma rüttelt am Status quo und bricht damit festgefahrene Strukturen auf - im Kontrast zum ,normalen' Bürger und in Auseinandersetzung mit dem Staat oder der Bürokratie (35, 27, 12, 8, 71, 23). So wird beispielsweise festgestellt, dass mit Jack Ma „[i]nmitten eines Volkes von Mitläufern Unternehmertum entstanden [ist]“ (8). Ähnlich dem Silicon Valley, das als ein „Mikrokosmos der außergewöhnlichen Persönlichkeiten“ (59) gilt, in dem mit „alternativen Regierungsmodellen“ (21) experimentiert wird, übernimmt auch Jack Ma die Rolle des dynamischen Gegenspielers zur trägen Bürokratie. So kontrastieren Alibabas Finanzdienstleistungen mit dem staatlich do- 
minierten Bankensektor, dem wenig kundenorientierte Dienstleistungen und ein Mangel an Innovationsbereitschaft angelastet werden (68).

Als Unternehmerheld vereint Jack Ma auf typische Weise generelle Unternehmereigenschaften mit einem individuellen, ja geradezu exzentrischen Charakter, wobei hier von einer bewussten Selbstinszenierung auszugehen ist, die von den Medien dankbar aufgenommen wird. Ma gilt als „eine der schillerndsten Unternehmerpersönlichkeiten“ (54) - vielleicht ein Grund, weshalb er in den deutschen Medien wesentlich größere Beachtung erfährt als seine Kollegen Robin Li (Baidu) oder Pony Ma (Tencent). Und es werden ihm „Charisma“" und „Präsenz“ zugeschrieben $(68,64)$. Er wird beschrieben als charmanter (29), humorvoller und selbstironischer „Exzentriker“ (49, 62, 54), der das Rampenlicht keineswegs scheut (39). Vielfach erwähnt sind seine Auftritte als Lady Gaga, Michael Jackson oder Punkrocker mit Irokesenschnitt, der zum Firmenjubiläum Karaoke singt (36, 39, 49). Deutsche Medien nennen Ma einen „Clown“ (75), englische sprechen von „crazy Jack“ (43). Auch sein Aussehen und sein selbstironischer Umgang hiermit wird bemerkt. Als Internetnutzer ihn mit der Figur des Gollum aus dem Herrn der Ringe oder einem Picasso-Gemälde verglichen, postete er das betreffende Bild und fragte: „Ist Picassos Malerei dermaßen gut oder liegt es daran, dass der dargestellte Mann so gut aussieht?" (39).

Die Darstellung Jack Mas als Unternehmerheld hat eine doppelte und auf den ersten Blick paradoxe Funktion: Er wird zum einen westlichen Vorbildern angepasst, die den Typus des Unternehmerhelden in reinster Form repräsentieren. Zum anderen steht er für China und den Erfolgsweg der chinesischen Entwicklung. Jack Ma wird damit dem Weltbild der Globalisten unterworfen, die in dem - doch nicht so fremden - China die besonders erfolgreiche Umsetzung des westlichen Weges erkennen, hier konkret: Innovation durch Akteure der Privatwirtschaft.

Die Parallelen des chinesischen Falles zu den Helden des Silicon Valley sind vielfach: Jack Ma wird als „Steve Jobs Asiens“ $(3,71,29)$ bezeichnet, sein Unternehmen als „chinesische[s] Amazon“ (68) und das Geschäftsmodell Alibabas als „chinesische[r] Mix aus Amazon, Ebay und Paypal“ (72). Ma selbst bedient diese Parallele mit dem Ausspruch: „Ebay mag ein Hai im Ozean sein, aber ich bin das Krokodil im Jangtse-Strom“ (37). Auch der bescheidene Anfang von Unternehmensgeschichten - bei Jack Ma in der „Firmenzentrale Wohnzimmer“ (49) und bei Steve Jobs in der Garage - wird erwähnt. Dieser Rückgriff auf westliche Äquivalente bietet westlichen Leserinnen und Lesern Verständnishilfen in Anbetracht eines eigentlich fremden, chinesischen Marktes. Umgekehrt liefert er einen weiteren Beweis der universalen Regel, dass Innovation durch privatwirtschaftliche Akteure vorangetrieben wird.

Gleichzeitig ist Jack Ma mehr als ein individueller Unternehmer mit heroischen Zügen: Er steht für das Unternehmen Alibaba (17), die gesamte Branche der chinesischen Start-up-Szene (47) und China schlechthin. „Ma’s rise“ spiegelt „China’s rise“ (25) wider, bzw. in der deutschen Variante: Er „verkörperte den chinesischen 
Aufstieg wie kein Zweiter" (44, siehe auch 13). Der Entwicklungsweg Chinas wird in dieser Parallelisierung greifbar, verliert aber seine Komplexität und Widersprüchlichkeit. Mit dem Start-up-Unternehmer Jack Ma als Gesicht und Sinnbild des chinesischen Wirtschaftswachstums und der chinesischen Innovationskraft treten die autoritären Züge des chinesischen Systems und die Eingriffe des Staates in das Wirtschaftsleben in den Hintergrund.

\section{Die Deheroisierung Jack Mas als Vertreter des chinesischen Staatskapitalismus}

Beruht die Heroisierung Jack Mas auf der Zuschreibung anscheinend universell ausmachbarer Unternehmereigenschaften, so wird er deheroisiert, indem diese in Frage gestellt werden. Dies geschieht vor allem, indem Ma in das System eingebettet und seine Abhängigkeit von der Regierung aufgezeigt wird. Überdies wird mit dem Hinweis auf Produktpiraterie Innovationsschwäche suggeriert, und es wird der Einfluss eines angeblichen Nationalcharakters geltend gemacht. Jack Ma wird so zum chinesischen Unternehmer, der ein System repräsentiert, welches sich von der westlichen Wirtschaftsordnung grundsätzlich unterscheidet, mit dieser konkurriert und aggressiv expandiert. Dabei nahmen negative Darstellungen Jack Mas in den vergangenen Jahren zu $(2,25,60,67,12,31,74)$, im Einklang mit der zunehmend kritischen Berichterstattung zu China und insbesondere als Reaktion auf Übernahmen deutscher (Traditions-)Unternehmen durch chinesische Betriebe.

Als chinesischer Unternehmer handelt Jack Ma nicht autonom, sondern in Kooperation mit und Abhängigkeit von der chinesischen Regierung, welche regulierend eingreift und einen „Unsicherheitsfaktor“ (47) für den Unternehmenserfolg darstellt (65). Das Verhältnis zwischen Staat und Unternehmen wird dabei unterschiedlich charakterisiert: Entweder sind chinesische Unternehmen wie Alibaba ein „Spielball der Politik“ (10), oder sie lehnen sich selbst gezielt an den Staat an und stellen sich mit diesem gut, um im chinesisch-autoritären Kapitalismus zu reüssieren und sich gegen die Risiken abzusichern, die vom Staat ausgehen $(2,25,12,65)$. Auch der Grad der Abhängigkeit vom Staat wird unterschiedlich bemessen: Die Position Jack Mas rangiert vom Partner des Staates $(44,10)$ über den Stellvertreter (10) und „inoffizielle[n] Gesandte[n] der chinesischen Regierung“ (75) bis hin zum „Chefdiplomaten“ (9). Deutschen Zeitungsberichten zufolge „kennen und schätzen" (10) sich Jack Ma und die Parteiführung, und Mas Auftritte werden im Staatsfernsehen übertragen (49). Er selbst gab 2014 auf die Frage, in welchem Verhältnis er zum chinesischen Staat stehe, die oft zitierte Antwort: „Fall in love with the government, but don't get married“ (74).

Jack Mas Nähe zum Staat gipfelt in seiner Parteimitgliedschaft, die 2018 bekannt wurde. Auch die Existenz von Parteizellen innerhalb des Unternehmens (2, $12,74,4)$ sorgte in den deutschen, vor allem aber in den US-Medien für wesentlich negativere Darstellungen des Unternehmers. So titelte die New York Times 2018 
ironisch: „Jack Ma, China's richest man belongs to the Communist Party. Of course.“ Im Text wurde erklärt: „Party membership provides a layer of protection in a country where private ownership protections are often haphazardly enforced or ignored entirely [...] The disclosure of Mr. Ma's membership reflects the thinking that the party controls the economy and society" (74). Eine Abhängigkeit zwischen Staat und Unternehmen wird schließlich auch in Bezug auf das geplante Sozialkreditsystem suggeriert, das in der deutschen Presse besonders negativ aufgenommen wurde (58). Hier wird unterstellt, dass der Staat auf die personenbezogenen Daten angewiesen ist, die ihm die Internetunternehmen liefern - welche ihrerseits für sie günstige Regularien erhoffen, die sie vor ausländischer Konkurrenz schützen.

Die zweite wichtige Strategie der Deheroisierung besteht in der Anlastung illegaler Praktiken, die Alibabas Erfolg nicht als Ergebnis von Innovation, sondern von Täuschung und Manipulation erscheinen lassen. Hierzu gehören das Kopieren erfolgreicher westlicher Produkte - die Plattform Alibaba sei ein „Paradies für Fälscher" (41) -, das Verfälschen der Umsatzzahlen (51), Steuerprobleme (7) sowie Ermittlungen gegen das Unternehmen in China selbst und in den USA wegen besagter Produktpiraterie. Diese Verfahren stürzen, so die Medien, das Geschäftsmodell Alibabas in die „größte Glaubwürdigkeitskrise seit seiner Gründung“ (7). Besondere Empörung löste dabei Mas Ausspruch aus, die Fälschungen seien den Originalen qualitativ und preislich überlegen $(26,61,41)$. Auch die Verletzung liberaler Normen, wie sie das westliche Wirtschaftssystem prägen, trägt zu einer negativen Wahrnehmung Jack Mas und Alibabas bei. Dazu zählen die Intransparenz des Börsengangs von Alibaba, die verschachtelte Unternehmensstruktur $(72,73)$ sowie die beschränkten Aktionärsrechte, die einer "freiwillige[n] Selbstenteignung“ (11) der Aktionäre gleichkomme, obwohl westliche Tech-Unternehmen gleichermaßen Gebrauch von diesen machen (11). Auch der Verweis auf negative Praktiken diskreditiert dabei nicht nur den Unternehmer und sein Unternehmen, sondern das ganze System: „Alibaba's problems are, in a very real sense, China’s problems“ (25).

Schließlich wird Jack Ma insofern deheroisiert, als ihm Einzigartigkeit und Individualität seiner Person aberkannt werden. Statt individueller Eigenschaften wird ihm ein klischeehaft gefasster Nationalcharakter attestiert: „Er denkt - typisch chinesisch - in ganz anderen Zeiträumen“ (38). Oder: „Angriff und Aggressivität gehören zu dem Charakter des Chinesen wie der Klick zum Internet“ (54) - eine Eigenschaft, die angeblich auch auf Jack Ma zutrifft. Strategische Planung (44, 72, 39) und Aggressivität stehen im Zentrum dieser negativen Zuschreibungen und ergänzen sich zu einem Bedrohungsszenario. In entsprechenden Texten findet sich eine stark ausgeprägte bellizistische Bildlichkeit $(4,48,72)$. Ma soll gesagt haben „Wir starten einen Krieg mit Ebay“ (38); „Alibaba greift an“ (51), ,arbeitet an der Eroberung des internationalen Markts“ (64) und stellt für Jack Ma einen „unverhoffte(n) Triumphzug“ (11) dar. Alibaba gehört für das Manager Magazin damit zu den ,an- 
griffslustigsten Unternehmen der Welt“. Mit der Verunsicherung einer westlichen Leserschaft spielend, titelt die Zeitschrift: „Wer hat Angst vor Jack Ma?“ und fragt: „Kann Europa gegen die Herausforderer bestehen?“ (72).

Mit der Charakterisierung Jack Mas als chinesischer Unternehmer wird nicht nur sein Status als Unternehmerheld untergraben - nicht Risikofreude, sondern Protektion erklärt seinen Erfolg -, sondern es wird ein Beispiel für die angebliche Andersartigkeit und Bedrohlichkeit Chinas aufgerufen. Die Deheroisierung der Person und die Diskreditierung des Systems verstärken sich gegenseitig und liefern in Zeiten zunehmender Systemkonkurrenz Argumente für ein unnachgiebiges Auftreten westlicher Regierungen gegenüber China.

\section{Schluss}

Die Darstellung Jack Mas in den deutschen Medien und die dazugehörigen Heroisierungs- und Deheroisierungsstrategien müssen im Kontext der aktuellen Spannungen (Handelskrieg zwischen China und den USA, Debatte um den IT-Konzern Huawei) und des globalen Konkurrenzkampfs um Innovationsführerschaft verstanden werden. In den Zeitungsberichten über Jack Ma geht es nicht um eine spezifische Unternehmer- und Unternehmensgeschichte, sondern um das Beispiel für übergeordnete Muster, die von unterschiedlichen Weltanschauungen unterfüttert sind, welche um Deutungshoheit über die gegenwärtige Konfliktlage ringen. Im positiven Fall wird die Innovationskraft Chinas anerkannt, und es werden solche Eigenschaften auf Jack Ma projiziert, die der westlichen Idealvorstellung des liberalen Kapitalismus entspringen. Hier verkörpert er den Unternehmerhelden Schumpeters, der sich durch Innovationsfreude, Risikobereitschaft und Tatendrang auszeichnet. Der einzige Unterschied zu den Unternehmerhelden des Silicon Valley liegt in seinem persönlichen Charakter. In dieser Ähnlichkeit wird dem ,Aufstieg' Mas, Alibabas und Chinas das Bedrohliche genommen. Im negativen Fall werden die angeblichen Eigenschaften des chinesischen Staatskapitalismus, wenn nicht sogar ein chinesischer Nationalcharakter auf Ma übertragen, die ihn zum chinesischen Unternehmer machen, der - typischerweise - mit dem Staat kooperiert, unlautere Geschäftspraktiken betreibt und zur Eroberung der Welt ansetzt. Wenige Texte bedienen diese beiden konträren Sichtweisen in reiner Form. Vielmehr weist die Darstellung Jack Mas dieselbe Ambivalenz auf, die auch das westliche China-Bild insgesamt kennzeichnet. Auch die Haltung zu Jack Ma changiert zwischen Bewunderung und Schrecken. 


\section{Quellenverzeichnis}

(1) Alibaba-Chef Jack Ma ist der reichste Chinese - auch ohne IPO, in: Handelsblatt, 1. September 2014, www.handelsblatt.com/finanzen/maerkte/ipo/alibaba-chef-jack-ma-ist-der-reich ste-chinese-auch-ohne-ipo-seite-2/10636850-2.html?ticket=ST-798855-4BgCIJnczZQke0Eagz pg-ap1, 11. Juli 2019.

(2) Alibaba Founder Jack Ma, China's Richest Man, Is Now Officially a Member of Its Communist Party, in: Fortune, 27. November 2018, fortune.com/2018/11/27/jack-ma-chinacommunist-party/, 11. Juli 2019.

(3) Alibaba-Gründer Jack Ma. Wie ein arbeitsloser Lehrer der Steve Jobs Asiens wurde, in: Stern, 19. September 2014, www.stern.de/wirtschaft/news/alibaba-gruender-jack-ma-wie-einarbeitsloser-lehrer-der-steve-jobs-asiens-wurde-3612956.html, 11. Juli 2019.

(4) Alibaba-Gründer. Milliardär Jack Ma ist Kommunist, in: Spiegel Online, 27. November 2018, www.spiegel.de/wirtschaft/unternehmen/alibaba-jack-ma-ist-mitglied-der-kommunisti schen-partei-a-1240693.html, 11. Juli 2019.

(5) Alibaba Gründer und Kultfigur. Bei Jack Ma lief's nicht immer rund, in: boerse.ard.de, 10. September 2017, boerse.ard.de/boersenwissen/boersengeschichte-n/bei-jack-ma-liefs-nicht -immer-rund100.html, 11. Juli 2019.

(6) Alibaba und die Suchmaschine, in: Tagesspiegel, 04. Oktober 2011, www.tagesspiegel.de/ wirtschaft/alibaba-und-die-suchmaschine/4685390.html, 11. Juli 2019.

(7) Hendrik Ankenbrand: Aktie im Blick. Alibabas Steuerproblem, in: Frankfurter Allgemeine Zeitung, 7. März 2015, www.faz.net/aktuell/finanzen/aktien/aktie-im-blick-alibabas-steuer problem-13468341.html, 11. Juli 2019.

(8) Hendrik Ankenbrand: Alibabas Erben. Eine Stadt auf dem Weg ins Internet, in: Frankfurter Allgemeine Zeitung, 22. März 2015, www.faz.net/aktuell/wirtschaft/netzwirtschaft/ali babas-erben-eine-stadt-auf-dem-weg-ins-internet-13489395.html, 11. Juli 2019.

(9) ders.: Alibaba-Gründer im Trump-Tower, in: Frankfurter Allgemeine Zeitung, 10. Januar 2017, www.faz.net/aktuell/wirtschaft/agenda/alibaba-verspricht-donald-trump-eine-millionarbeitsplaetze-14612472.html, 11. Juli 2019.

(10) ders.: China. Alibabas böses Erwachen, in: Frankfurter Allgemeine Zeitung, 2. Februar 2015, www.faz.net/aktuell/wirtschaft/agenda/alibabas-boeses-erwachen-13403866.html, 11. Juli 2019.

(11) Hendrik Ankenbrand / Norbert Kuls: Neuer Star der Wall Street. Jack Mas unverhoffter Triumph mit Alibaba, in: Frankfurter Allgemeine Zeitung, 18. September 2014, www.faz. net/aktuell/finanzen/aktien/neuer-star-der-wall-street-jack-mas-unverhoffter-triumphzug-mit -alibaba-13158972.html, 11. Juli 2019.

(12) David Barboza: The Jack Ma Way. At Alibaba, the Founder is Squarely in Charge, in: The New York Times, 6. September 2014, www.nytimes.com/2014/09/07/business/internatio nal/at-alibaba-the-founder-is-squarely-in-charge.html, 11. Juli 2019.

(13) Georg Blume: Alibabas verborgener Schatz, in: Spiegel Online, 15. September 2018, www.spiegel.de/wirtschaft/unternehmen/daniel-zhang-alibabas-verborgener-schatz-a-1228 234.html, 11. Juli 2019. 
(14) Börsengang mit Weltrekord. Alibaba stellt Facebook in den Schatten, in: Handelsblatt, 22. September 2014, www.handelsblatt.com/finanzen/maerkte/aktien/boersengang-mit-welt rekord-alibaba-stellt-facebook-in-den-schatten/10735084.html, 11. Juli 2019.

(15) Jannis Brühl: Überwachung. China, Orwell und die Angst des Westens, in: Süddeutsche Zeitung, 11. Mai 2019, www.sueddeutsche.de/digital/china-kredit-sesame-sozialkredit-ueber wachung-1.4442172, 11. Juli 2019.

(16) Clay Chandler u. a.: China's Web King, in: Fortune International (Europe) 156.11, 10. Dezember 2007, S. 52-57.

(17) Chinesischer Internet-Gigant. Alibaba-Chef $\mathrm{Ma}$ will Bill Gates von Asien werden, in: Frankfurter Allgemeine Zeitung, 8. September 2018, www.faz.net/aktuell/wirtschaft/ unternehmen/online-handel-alibaba-chef-ma-kuendigt-seinen-rueckzug-aus-konzern-an-15 777558.html, 11. Juli 2019.

(18) Das „Krokodil vom Jangtse“, in: Frankfurter Allgemeine Zeitung, 6. September 2014, www.faz.net/aktuell/wirtschaft/menschen-wirtschaft/alibaba-chef-jack-ma-das-krokodil-vom -jangtse-13138801.html, 11. Juli 2019.

(19) Der reichste Chinese verdiente im Online Handel Milliarden, in: Manager Magazin, 10. Oktober 2018, www.manager-magazin.de/unternehmen/handel/ranking-der-reichstenchinesen-alibaba-gruender-jack-ma-wieder-auf-platz-1-a-1232479.html, 11. Juli 2019.

(20) Die Skepsis gegenüber den Investoren aus China wächst, in: Handelsblatt, 28. November 2018, www.handelsblatt.com/unternehmen/industrie/firmenuebernahmen-die-skepsis-gegen ueber-den-investoren-aus-china-waechst/23686100.html, 11. Juli 2019.

(21) Die vereinigten Staaten von Google, in: Zeit Online 33, 2014, www.zeit.de/2014/33/ suchmaschine-google-zukunft/seite-4, 11. Juli 2019.

(22) Die wichtigsten Denker der Welt ermittelt, in: Organisator, 21. Dezember 2015, www. organisator.ch/die-wichtigsten-denker-der-welt-ermittelt/, 12. Juli 2019.

(23) Rengachary Dinakaran: Jack Ma Says ,New Manufacturing“ Will Disrupt Global Manufacturing Industry, in: The Hindu Business Line, 19. September 2018, www.thehindu businessline.com/info-tech/new-manufacturing-will-disrupt-global-manufacturing-adapt-or -disappear-says-jack-ma/article24984867.ece, 12. Juli 2019.

(24) Axel Dorloff: Sozialkredit-System. China auf dem Weg in die IT-Diktatur, in: Deutschlandfunk, 23. Juni 2018, www.deutschlandfunk.de/sozialkredit-system-china-auf-dem-wegin-die-it-diktatur.724.de.html?dram:article_id=421115, 12. Juli 2019.

(25) Gady Epstein: The Face of China Inc, in: Forbes, 11. April 2011, S. 87-91.

(26) Fälschungen besser als das Original. Alibaba-Gründer Jack Ma, in: Spiegel Online, 15. Juni 2016, www.spiegel.de/wirtschaft/unternehmen/jack-ma-von-alibaba-haelt-faelschungen-fuerbesser-als-das-original-a-1097857.html, 12. Juli 2019.

(27) Rebecca Fannin: How I Did It. Jack Ma. Alibaba.com, in: Inc. Magazine, Januar 2008, S. 104-106.

(28) Martin Farrer: US-China Trade Skirmishes Obscure the Start of Tech Cold War, in: The Guardian, 21. Mai 2019, www.theguardian.com/business/2019/may/21/huawei-analysisus-china-trade-skirmishes-obscure-the-start-of-tech-cold-war, 12. Juli 2019. 
(29) Robert Gast: Dokumentation über Alibaba-Börsengang. Film drehen statt steinreich werden, in: Süddeutsche Zeitung, 12. Mai 2014, www.sueddeutsche.de/digital/dokumen tation-ueber-alibaba-boersengang-film-drehen-statt-reich-werden-1.1958472, 12. Juli 2019.

(30) Christoph Giesen: Kapitalismus. China im Zweikampf der Systeme, in: Süddeutsche Zeitung, 4. November 2016, www.sueddeutsche.de/wirtschaft/samstagsessay-zweikampf-dersysteme-1.3234649, 12. Juli 2019.

(31) Neil Gough / Alexandra Stevenson: The Unlikely Ascent of Jack Ma, Alibaba's Founder, in: The New York Times, 7. Mai 2014, www.nytimes.com/2014/05/08/technology/theunlikely-ascent-of-jack-ma-alibabas-founder.html, 12. Juli 2019.

(32) Ulrich Groothius: Wenn Alibaba-Chef Jack Ma eine Aktie wäre, in: WirtschaftsWoche 38, 14. September 2018, S. 23.

(33) Angela Gruber: Chinas Social Credit System. Volle Kontrolle, in: Spiegel Online, 28. Dezember 2017, www.spiegel.de/netzwelt/netzpolitik/china-social-credit-system-einpunktekonto-sie-alle-zu-kontrollieren-a-1185313.html, 12. Juli 2019.

(34) Gründer Jack Ma gibt Alibaba-Chefposten auf, in: Spiegel Online, 8. September 2018, www.spiegel.de/wirtschaft/unternehmen/alibaba-gruender-jack-ma-verlaesst-chinesischenonline-konzern-a-1227157.html, 12. Juli 2019.

(35) Florian Günther: Jack Ma - der reichste Mann Asiens, in: Investorenausbildung, ohne Datum, investorenausbildung.de/jack-ma-der-reichste-mann-asiens/, 12. Juli 2019.

(36) Niclas Hagen: Die Alibaba-Story, in: StartingUp: das Gründermagazin, ohne Datum, www.starting-up.de/geschaeftsideen/unternehmer-typen/die-alibaba-story.html, 12. Juli 2019.

(37) Lise Hegemann: Roadshow des Online-Riesen. Alibaba trommelt für Rekordbörsengang, in: Handelsblatt, 8. September 2014, www.handelsblatt.com/finanzen/maerkte/ipo/road show-des-online-riesen-alibaba-trommelt-fuer-rekordboersengang/10658566.html?ticket= ST-2341753-YqXoz4eej5CuzcQEpzjj-ap1, 12. Juli 2019.

(38) Wolfgang Hirn: Alibaba-Gründer Jack Ma. Der Mann, der Ebay besiegte, in: Manager Magazin, 22. Mai 2015, www.manager-magazin.de/unternehmen/handel/alibaba-gruen der-jack-ma-der-mann-der-ebay-besiegte-a-1035056.html, 12. Juli 2019.

(39) Sha Hua: Weltgeschichte. Papa Jack Ma, in: Handelsblatt, 17. November 2017, www. handelsblatt.com/politik/international/weltgeschichten/hua/weltgeschichte-papa-jack-ma/ 20598220.html, 12. Juli 2019.

(40) Internet-Konzern. Yahoo verdient Milliarden dank Alibaba, in: Süddeutsche Zeitung, 22. Oktober 2014, www.sueddeutsche.de/wirtschaft/internet-konzern-yahoo-verdient-milliardendank-alibaba-1.2185286, 12. Juli 2019.

(41) Jack Ma. Alibaba-Gründer: Plagiate sind oft besser als Originale, in: Frankfurter Allgemeine Zeitung, 15. Juni 2016, www.faz.net/aktuell/wirtschaft/netzwirtschaft/alibaba-gruen der-jack-ma-findet-plagiate-besser-als-originale-14288569.html, 12. Juli 2019.

(42) Jack Ma. The Man Leading Alibaba to America, in: Time, 6. Oktober 2014, S. 18.

(43) Christian Koch: Lead Like... Jack Ma, in: Director 72.2, November 2018, S. 28.

(44) Florian Kolf / Stephan Scheuer: Alibaba-Chef Jack Ma treibt seinen Angriff auf den globalen Onlinehandel voran, in: Handelsblatt, 2. Januar 2019, www.handelsblatt.com/unter 
nehmen/handel-konsumgueter/e-commerce-alibaba-chef-jack-ma-treibt-seinen-angriff-aufden-globalen-onlinehandel-voran/23819996.html, 12. Juli 2019.

(45) Stefan Kornelius: China - Europa. Notwendige Rivalität, in: Süddeutsche Zeitung, 26. März 2019.

(46) Luisa Kroll / Kerry A. Dolan: Billionaires. The Richest People in the World. \#21 Jack Ma, in: Forbes, 5. März 2019, www.forbes.com/profile/jack-ma/\#1ea5139f1ee4, 12. Juli 2019.

(47) Johannes Kuhn: Alibaba-Börsengang. Chinas Amazon könnte den größten Börsengang aller Zeiten schaffen, in: Süddeutsche Zeitung, 7. Mai 2014, www.sueddeutsche.de/wirt schaft/alibaba-boersengang-chinas-amazon-koennte-den-groessten-boersengang-aller-zei ten-schaffen-1.1953261, 12. Juli 2019.

(48) Adam Lashinsky: Ma vs. Ma, in: Fortune, 1. Juli 2018, S. 73-83.

(49) Felix Lee: Alibaba-Chef Jack Ma. Ein bisschen Exzentriker, in: Zeit Online, 10. September 2014, https://www.zeit.de/wirtschaft/unternehmen/2014-09/alibaba-jack-ma, abgerufen am 12. Juli 2019.

(50) ders.: Social Scoring in China. Im Reich der überwachten Schritte, in: taz, 10. Februar 2018, taz.de/Social-Scoring-in-China/!5480926/, 12. Juli 2019.

(51) ders.: Onlinehandel. Alibaba greift an, in: Zeit Online, 17. September 2012, www.zeit. de/wirtschaft/2012-09/alibaba-china, 12. Juli 2019.

(52) Jörg Lichter: Chinas neue Seidenstraße. Das Versagen des Westens, in: Handelsblatt, 1. März 2019, www.handelsblatt.com/politik/konjunktur/research-institute/hri-analyse-chi nas-neue-seidenstrasse-das-versagen-des-westens/24051100.html, 12. Juli 2019.

(53) Ma und das Mehr, in: Euro am Sonntag 39, 27. September 2014, S. $26-27$.

(54) Philipp Mattheis: Der Alibaba-Gründer im Portrait. Die fünf Gesichter des Jack Ma, in: WirtschaftsWoche, 19. September 2014, www.wiwo.de/unternehmen/handel/der-alibabagruender-im-portraet-die-fuenf-gesichter-des-jack-ma/10690782.html, 12. Juli 2019.

(55) Philipp Mattheis / Stephan Happel: Jack Ma. Wie ein Visionär Alibaba zum Erfolg führte, in: WirtschaftsWoche, 25. September 2013, aktualisiert 8. September 2014, www.wiwo.de/ unternehmen/handel/jack-ma-wie-ein-visionaer-alibaba-zum-erfolg-fuehrte/8784144.html, 12. Juli 2019.

(56) Milliardär des Monats. Jack Ma, in: Euro 5, 23. April 2014, S. 14.

(57) Danielle Paquette: Jack Ma Scraps his Pledge to Create 1 Million U.S. Jobs. But Was it Ever Serious?, in: The Washington Post, 20. September 2018, www.washingtonpost.com/ world/jack-ma-withdraws-his-pledge-for-a-million-us-jobs-analysts-say-that-was-a-pipe -dream-anyway/2018/09/20/84da5cee-bc8b-11e8-97f6-0cbdd4d9270e_story.html?noredirect $=$ on\&utm_term=.00aab4af0c77, 12. Juli 2019.

(58) Stephan Scheuer: Unternehmer mit Parteibuch. Alibaba-Gründer Jack Ma ist Chinas reichster Kommunist, in: Handelsblatt, 28. November 2018, www.handelsblatt.com/unter nehmen/management/unternehmer-mit-parteibuch-alibaba-gruender-jack-ma-ist-chinas-reich ster-kommunist/23690808.html, 12. Juli 2019.

(59) Thomas Schulz: Silicon-Valley-Forschung zu Langlebigkeit. Eine Zukunft, in der Altern nicht schmerzt, in: Spiegel Online, 19. August 2018, www.spiegel.de/gesundheit/diagno se/silicon-valley-forscher-wollen-das-altern-aufhalten-a-1223170.html, 12. Juli 2019. 
(60) Michael Schuman: Alibaba and the 40,000 Thieves, in: Forbes, 23. November 2015, S. $100-124$.

(61) Lukas Schürmann: Für Jack Ma sind Fälschungen aus China besser als die Originale, in: Manager Magazin, 15. Juni 2016, https://www.manager-magazin.de/unternehmen/han del/alibaba-chef-jack-ma-china-faelschungen-besser-als-originale-a-1097732.html, 12. Juli 2019.

(62) Christopher Scott: New Report Shows China Closing Innovation Gap, in: Asia Times, 8. April 2019, www.asiatimes.com/2019/04/article/new-report-shows-chinas-innovation-out pacing-us/, 12. Juli 2019.

(63) Mark Siemons: Chinas Sozialkreditsystem. Die totale Kontrolle, in: Frankfurter Allgemeine Zeitung, 11. Mai 2018, www.faz.net/aktuell/feuilleton/debatten/chinas-sozialkredit system-die-totale-kontrolle-15575861.html, 12. Juli 2019.

(64) Mark Siemons: Der Internetriese Alibaba. Wo die chinesischen Träume leben, in: Frankfurter Allgemeine Zeitung, 13. Mai 2014, www.faz.net/aktuell/feuilleton/internetriese-ali baba-dieser-laden-ist-groesser-als-ebay-und-amazon-zusammen-12935623.html, 12. Juli 2019.

(65) Mark Siemons: Internetkonzern Alibaba an der Börse. Und die wollen Amazon Paroli bieten?, in: Frankfurter Allgemeine Zeitung, 22. September 2014, www.faz.net/aktuell/ feuilleton/debatten/alibaba-an-der-boerse-konkurrenz-fuer-amazon-13165613.html, 12. Juli 2019.

(66) Frank Sieren: Sierens China: Jack Ma - Ein Visionär auf dem Weg zur Tür, in: Deutsche Welle, 12. September 2018, www.dw.com/de/sierens-china-jack-ma-ein-vision\%C3\%A4r-aufdem-weg-zur-t\%03\%BCr/a-45467015-0, 12. Juli 2019.

(67) Pete Sweeney: Jack Ma's American Dream Runs Up Against China First, in: The New York Times, 20. September 2018, www.nytimes.com/2018/09/20/business/dealbook/jackmas-american-dream-runs-up-against-china-first.html, 12. Juli 2019.

(68) Nina Trentmann: Dieser Ex-Lehrer ist der Schreck von Amazon, in: Die Welt, 22. April 2014, www.welt.de/wirtschaft/article127200039/Dieser-Ex-Lehrer-ist-der-Schreck-von-Ama zon.html, 12. Juli 2019.

(69) Unbegrenzte Amtszeit für Xi Jinping, in: Zeit Online, 11. März 2018, www.zeit. $\mathrm{de} /$ politik/ausland/2018-03/nationaler-volkskongress-xi-jinping-china-unbegrenzte-amtszeit, 12. Juli 2019.

(70) Vom Arbeitslosen zum Milliardär. Alibaba-Gründer Jack Ma ist reichster Asiate, in: ntv, 12. Dezember 2014, www.n-tv.de/wirtschaft/Alibaba-Gruender-Jack-Ma-ist-reichster-Asiatearticle14143066.html, 12. Juli 2019.

(71) Bettina Weiguny: Jack Ma auf der Cebit. Ein Weltstar in Hannover, in: Frankfurter Allgemeine Zeitung, 15. März 2015, www.faz.net/aktuell/wirtschaft/cebit/alibaba-chef-jackma-eroeffnet-die-cebit-2015-13484202.html, 12. Juli 2019.

(72) Wer hat Angst vor Jack Ma?, in: Manager Magazin 4, 20. März 2015, S. 30.

(73) Li Yuan: Alibaba's Jack Ma, China's Richest Man, to Retire From Company he CoFounded, in: The New York Times, 7. September 2018, www.nytimes.com/2018/09/07/ technology/alibaba-jack-ma-retiring.html, 12. Juli 2019. 
(74) ders.: Jack Ma, China's Richest Man, Belongs to the Communist Party. Of Course, in: The New York Times, 27. November 2018, www.nytimes.com/2018/11/27/business/jackma-communist-party-alibaba.html, 12. Juli 2019.

(75) Bernhard Zand: Der Clown tritt ab, in: Spiegel Online, 8. September 2018, www.spiegel. de/wirtschaft/unternehmen/jack-ma-zieht-sich-bei-alibaba-zurueck-der-clown-tritt-ab-a-122 7176.html, 12. Juli 2019. 